\title{
Melting and Reentrant Freezing of Two-Dimensional Colloidal Crystals in Confined Geometry
}

\author{
R. Bubeck, C. Bechinger, S. Neser, and P. Leiderer \\ Faculty of Physics, University of Konstanz, D-78457 Konstanz, Germany
}

(Received 25 November 1998)

\begin{abstract}
We study the melting behavior of a finite number $(N \leq 45)$ of paramagnetic colloidal spheres in a two-dimensional circular hard wall cavity. The interaction strength between the particles is varied by applying a magnetic field $B$. At high $B$, i.e., strong interaction, the particles are arranged in a highly ordered shell-like structure. With decreasing $B$ we observe first a loss of angular order between adjacent shells. Upon further reduction of the external $B$ field, however, angular order is restored again before the system melts completely. We propose a simple mechanism to account for this reentrance phenomenon. [S0031-9007(99)08972-3]

PACS numbers: 82.70.Dd, 64.60.Cn, 83.20.Hn
\end{abstract}

During the past decade there has been considerable progress in the localization and cooling of ions and electrons in artificial confining fields. Typical examples for three-dimensional (3D) and two-dimensional (2D) systems are ions in radio frequency traps [1], electrons on the surface of liquid $\mathrm{He}[2,3]$, and electrons in quantum dots [4], respectively. With the help of present-day powerful imaging techniques such examples may be promising subjects for the experimental investigation of systems in lateral confinements. Additionally the structural and dynamical properties of few-body systems are also attractive from the theoretical point of view. Several authors considered 2D systems with finite numbers of ions or electrons in lateral confinements using Monte Carlo (MC) simulations [5-9]. At low temperatures and in the case of a small number of particles, the clusters are found not to crystallize in a triangular lattice (Wigner crystal), but are arranged in a shell structure. Accordingly, it was pointed out that such systems may be a "realization" of a 2D Thomson atom where the structure as a function of the particle number can be analyzed in terms of a Mendeleev-type table [7]. The melting of laterally confined 2D systems with particle numbers on the order of 100 or smaller is theoretically predicted to occur via a two step process [7-9]. Upon increasing the temperature, first intershell rotation becomes possible where orientational order between adjacent shells is lost. At even higher temperatures radial diffusion between shells sets in which finally destroys positional order. This scenario differs considerably from what is generally predicted to occur in infinite 2D systems when the temperature is raised [10-13].

In this paper we present an experimental study of the phase behavior of a 2D system in a circular hard wall confinement. As particles we used superparamagnetic colloidal spheres whose pair potential can be varied over a wide range by an external magnetic field $B$. The advantages of colloidal suspensions as model systems are their convenient time (milliseconds) and length scales (microns) which allow the detailed observation of single particle trajectories by means of video microscopy $[14,15]$. At high
$B$, i.e., strongly repulsive forces between the particles, we observe a shell structure displaying radial and orientational order. With decreasing $B$ we observe first a loss of angular order between adjacent shells. Upon further reduction of the external $B$ field, however, angular order is restored again before the system melts completely. We propose a simple mechanism to account for this reentrance phenomenon.

As substrate for the 2D colloidal system we used fused silica plates onto which a 3-4 $\mu \mathrm{m}$ thick smooth film of poly(methyl-methacrylate) (PMMA) was deposited by spin coating. This was necessary to prevent the particles from sticking to the surface. The lateral confinement was realized by a TEM (transmission electron microscope) grid made of copper and a thickness of $15 \mu \mathrm{m}$ which was heated to about $150{ }^{\circ} \mathrm{C}$ and then pressed into the thin PMMA film. After this process we obtained typically several tens of identical circular compartments (diameter $72 \mu \mathrm{m}$ ) with perpendicular walls (Fig. 1a). For the details of the sample preparation we refer to the literature [15]. After insertion of the superparamagnetic colloidal spheres which were suspended in water (DynaBeads $4.5 \mu \mathrm{m}, \mathrm{Fa}$. Dynal, Lot-No. A44610) and stabilized with $0.2 \mathrm{wt} \%$ Triton X-100, the particles sedimented towards the shaped bottom plate where they formed a 2D colloidal system in each compartment (Fig. 1b). The number density in different compartments varied statistically between about 25 and 45 particles at the concentrations used in our experiments. Another silica plate was mounted on top of a)

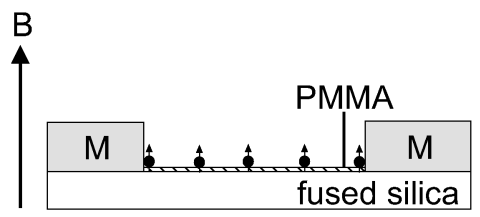

b)

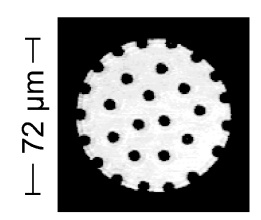

FIG. 1. Schematic side view of the experimental cell (a). A TEM mesh (M) serves as lateral confinements for the particles. Photograph (top view) of a single compartment which is occupied by a 2D colloidal system (b). 
the bottom plate with a $1 \mathrm{~mm}$ spacer in between to avoid contamination of the suspension with dust. The cell was placed in the center of a copper coil, the latter producing magnetic fields of up to $2 \mathrm{mT}$ which induced a magnetic moment $M$ in the particles leading to a repulsive magnetic dipole pair potential $V_{i, j}^{\mathrm{mag}}=\mu_{0} M^{2} / 4 \pi r_{i, j}^{3}$, where $r_{i, j}$ is the particle distance $[15,16]$. The colloidal spheres were imaged with a home-built inverted video microscope onto a CCD camera and the data were stored on videotape. With a particle recognition algorithm we obtained the trajectories of the spheres with a lateral and temporal resolution of $0.25 \mu \mathrm{m}$ and $20 \mathrm{~ms}$, respectively. Prior to each measurement the system was allowed to equilibrate for several hours. This was necessary due to the small lateral diffusion constant of the particles $D=0.35 \mu \mathrm{m}^{2} / \mathrm{s}$. At the beginning of each run the cell was carefully adjusted horizontally because a tilted substrate would lead to a superimposed drift motion (manifesting itself in a quadratic contribution to the measured mean square displacement) and to gradients in the particle density.

To describe the effective interaction between the paramagnetic spheres it is useful to introduce the dimensionless plasma parameter $\Gamma=\left\langle V^{\mathrm{mag}}\right\rangle / N k_{B} T$, where the brackets correspond to the sum over all pairs $i, j, N$ to the number of particles and $k_{B} T$ to the thermal energy. Since $B$ plays the role of an inverse effective temperature which can be adjusted precisely by the external magnetic field, during our experiments we varied $B$, whereas the temperature was kept constant at $T=295 \mathrm{~K}$. In Figs. $2 \mathrm{a}-2 \mathrm{~d}$ we present exemplary the $\Gamma$ dependence of the trajectories measured over $30 \mathrm{~min}$ each for a system consisting of $N=$ 29 particles. We want to emphasize, however, that a simi- a)
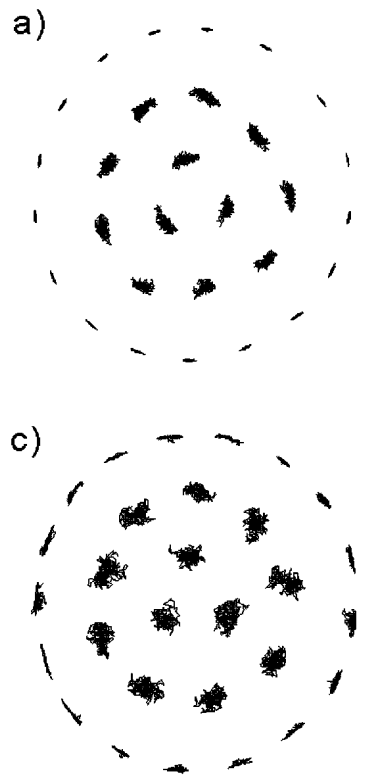

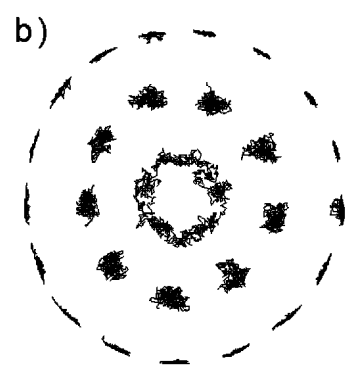

d)

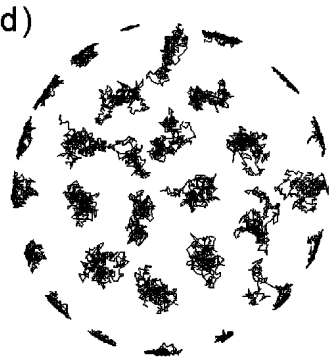

FIG. 2. Particle trajectories $(N=29)$ in a circular confinement for decreasing $\Gamma$ : (a) 152; (b) 38; (c) 30; (d) 7.5. The data in each picture represent runs of $30 \mathrm{~min}$. lar sequence as discussed below is also observed for, e.g., $N=34,37$, and 40 , though the shell number which starts to rotate first strongly depends on the particle configuration. Therefore it seems that the behavior is caused by the finiteness of the investigated system and not by a particular particle number. In Fig. 2 a which corresponds to a plasma parameter $\Gamma=152$ the particles are localized in shells with an average interparticle distance which depends both on the radius (shell number) and the total number of particles. In the case shown here, the particles are arranged in three equally spaced shells consisting of 3,9 , and 17 particles, respectively. Most particles of the inner two rings are sixfold coordinated, whereas the number of next neighbors of the edge particles is typically smaller. To study the motion of the particles comprising the first shell, we plotted their time-dependent angular positions $\phi_{i}(i=1,2,3)$ together with the corresponding mean value as dashed and solid lines, respectively (Fig. 3a). Obviously the angular positions of the different particles are not independent of each other but rather are highly correlated, i.e., the shell can be considered as rigid for $\Gamma=152$. To analyze the
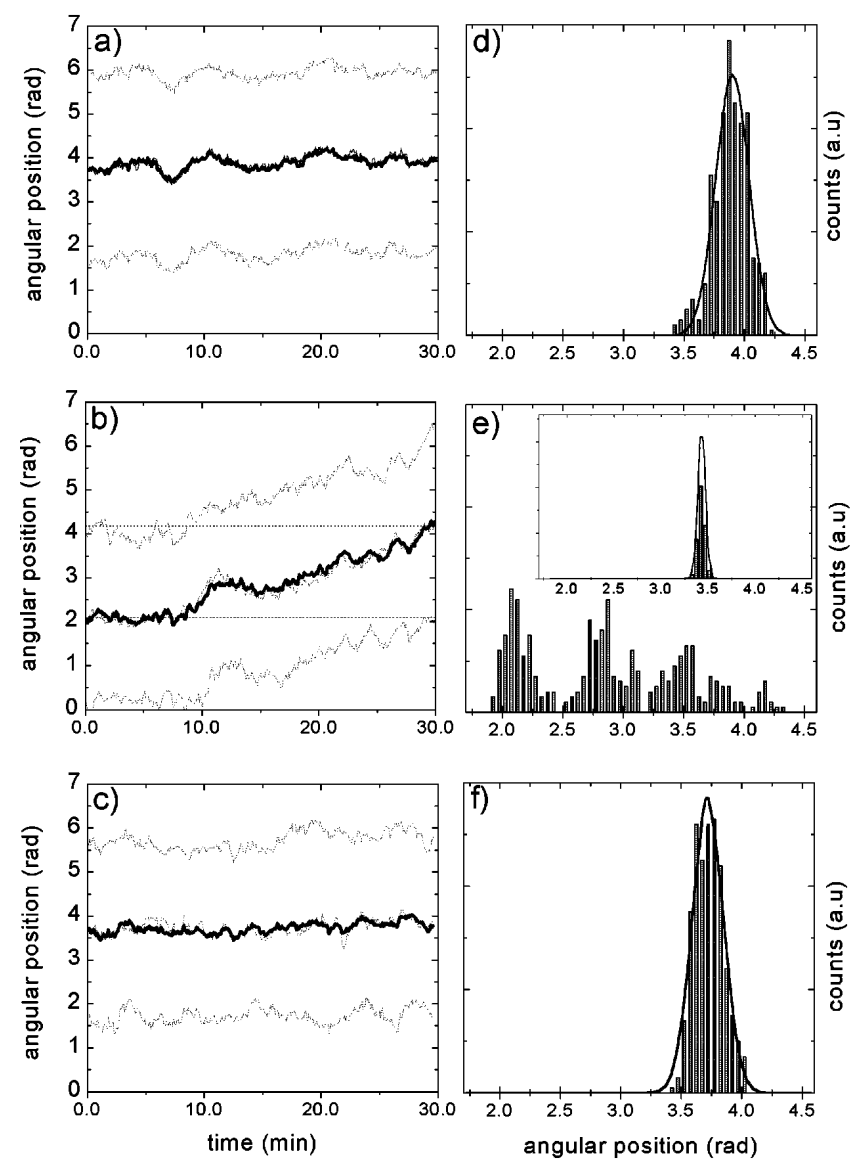

FIG. 3. Left side: Temporal behavior of the angular positions of the three particles (thin lines) comprising the first shell at $\Gamma=152, \Gamma=38$, and $\Gamma=30$; (a), (b), and (c), respectively. The thicker lines are the time-dependent mean values. Right side: Corresponding histograms of the mean values [(d)-(f)]; the inset of (e) additionally shows the data for the second shell. 
angular movement of the whole shell, we calculated the histogram of the mean value which can be described by a Gaussian curve, the half-width corresponding to about $12 \%$ of the angular interparticle distance (Fig. 3b). Similar results were also found for the radial and angular particle distributions of the other shells, which suggests that the colloidal spheres in Fig. 2a are localized around equilibrium positions (only the angular distribution of the outmost ring shows a slightly asymmetric shape due to unbalanced forces which push those particles against the hard wall). When we additionally computed particle density profiles by MC simulations [15], at low temperatures we found exactly the same shell structure as shown in Fig. 2a. Therefore this configuration can be considered as the ground state. For very large numbers of MC steps the system can also rotate as a whole. This, however, was not observed on the time scale of our experiments due to the slow dynamics of the colloidal spheres. It should be noted that shell structures comparable to Fig. 2a are also observed in theoretical studies of electrons in hard wall confinements [8] or parabolic potentials $[5,6,7,9]$ at low temperatures and can be explained by a competition between two different types of ordering: while an infinitely large 2D system of repulsively interacting particles would energetically favor a triangular arrangement, the shell structure is imposed by the circular symmetry of the confinement which apparently dominates the structure.

Figure $2 \mathrm{~b}$ shows the particle trajectories at $\Gamma=38$ which corresponds to a lower magnetic field. In contrast to $\Gamma=152$ where all the particles are localized at welldefined equilibrium positions here this is no longer the case for the colloids in the first shell. Their trajectories are not separated in space, but do overlap and are smeared out into a ring during our measuring time. A comparison of the time-dependent angular particle positions reveals that the three particles still perform an essentially collective motion which is only slightly disturbed by independent Brownian fluctuations (Fig. 3b). The angle of the collective excursion which is covered during $30 \mathrm{~min}$ is now $135^{\circ}$, i.e., the particles in the first shell are able to permute their angular positions and diffuse along the shell. This is also obvious from the histogram in Fig. 3e which is not Gaussian anymore but shows several peaks. The histogram of the second shell, however, which is plotted as an inset in Fig. 3e, is still a narrow Gaussian curve and clearly demonstrates that the first shell rotates relative to the other particles at $\Gamma=38$. We will refer to such relative collective rotations as intershell rotation.

Intershell rotation is also known from theoretical studies of electron clusters in lateral confinements and thus seems to not be limited to a particular pair interaction potential $[5,6,7,9]$. From a normal mode analysis of the excitation spectrum of electron clusters, a very low eigenfrequency is obtained which is attributed to a collective motion of particles within a shell. When the particle numbers of adjacent shells are a multiple of each other this leads to a registration of neighboring shells and thus to high restoring forces upon shell distortions. Otherwise the energy barrier for intershell rotation $\Delta E$ may be much smaller [9]. Consequently the shell where intershell rotation is observed first strongly depends on the particle configurations, i.e., the total number of particles [7,8]. For $N=40$, e.g., we find the second out of four shells to rotate first.

Upon further reduction of $\Gamma$, at first glance one would expect the shells to overcome $\Delta E$ more easily, i.e., intershell rotation to be more facilitated. This, however, is in contradiction to our experimental results. The trajectories plotted in Fig. 2c clearly demonstrate that intershell rotation disappears at $\Gamma=30$ and orientational order is reestablished again. This is also supported by Figs. 3c and 3f which show essentially the same behavior as the corresponding plots at $\Gamma=152$. We want to emphasize that such a reentrant ordered state has not been observed in computer simulations on two-dimensional Coulomb clusters $[7,8]$. As will be discussed in detail further, this reoccurrence of angular order at smaller $\Gamma$ can be explained by correlation effects between angular and radial fluctuations of the particles.

When the magnetic field is decreased further, the repelling forces between the spheres finally become so weak that both positional and orientational order is lost and the cluster melts completely. This can be seen in Fig. 2d $(\Gamma=7.5)$ where the shell structure is hardly visible any more and some of the edge particles perform excursions even towards the center of the compartment.

We performed measurements at 14 different magnetic fields and analyzed the trajectories as mentioned above to distinguish ordered states from intershell rotation. From those data we obtained a phase sequence which is plotted in Fig. 4a. With decreasing $\Gamma$ we observe the sequence: angular and radial order-intershell rotation-angular and radial order-liquid (the latter denoting the absence of a shell structure).

In contrast to infinite $2 \mathrm{D}$ systems, where melting is often discussed to occur via a two step process, where first translational and only then orientational order disappears, here the melting process is rather different. As a result of the lateral confinement the particles are arranged in a shell structure which is - due to the strongly distance-dependent interaction potential - very stable regarding loss of radial order. In contrast, angular reorientations between adjacent shells require only small changes in the relative particle distances and are thus energetically favored. Accordingly, the motion of particles at high $\Gamma$ is highly anisotropic which can be seen in Fig. $2 \mathrm{a}$ and becomes most pronounced at $\Gamma=38$ where intershell rotation occurs. With decreasing $\Gamma$, however, also the strength of radial particle fluctuations grows as can be seen by the increase of the radial particle excursions in the trajectory plots (cf. Figs. $2 a-2 d$ ). Figure $4 b$ shows the half-width of the radial distribution of the particles in the first and second shell which increases more or less monotonically with 
a)

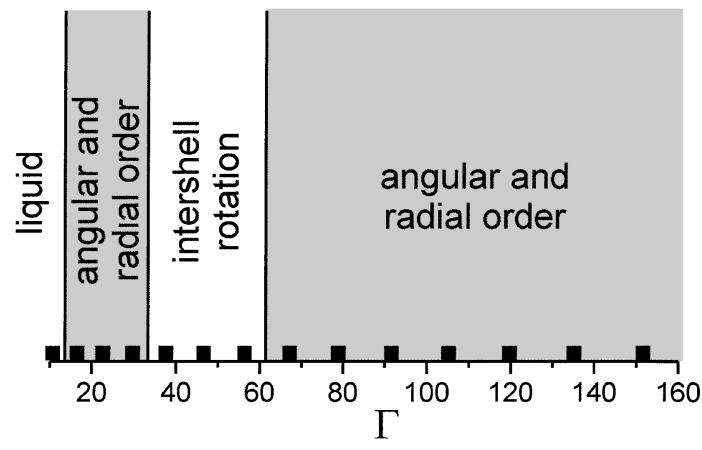

b)

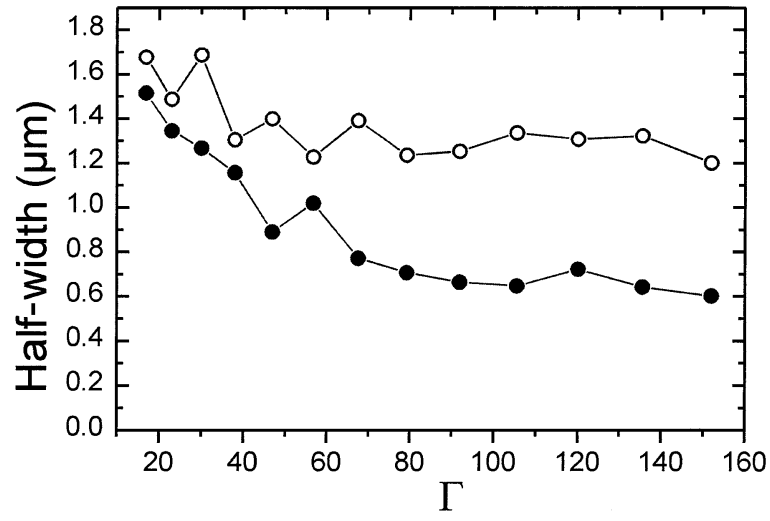

FIG. 4. (a) Sequence of states for a system consisting of $N=$ 29 particles as a function of the plasma parameter. The plot is obtained from 14 data points (symbols) between $\Gamma=152$ and 7.5. (b) Half-width of the radial extension of the first $(-)$ and second shell $(\bigcirc)$ which increases as $\Gamma$ is reduced.

decreasing $\Gamma$. Accordingly, the energy barrier for particles in adjacent shells to pass each other increases and finally leads to the disappearance of intershell rotation as observed in our experiments. For even smaller $\Gamma$ the radial fluctuations destroy the shell structure and the cluster melts completely.

It is an interesting question why such a reentrance phenomenon was not observed in simulations of electronic clusters in lateral confinements. In addition to the different pair interaction potential and the presence of hydrodynamic interactions, it is likely that the details of the boundary condition which determine to what extent the particles can escape in a lateral direction upon changing $\Gamma$ are important. Since most of the calculations on electron clusters are performed in the presence of a parabolic confining potential, the density in the system, i.e., the coupling between particles, varies as a function of $\Gamma$. This may also have consequences in the melting scenario of such systems.

In summary, we have studied the melting of a twodimensional system of superparamagnetic particles in a circular hard wall confinement. For the small particle numbers used in our experiments we found the particles to be arranged in a shell structure due to the boundary conditions. Upon reducing the plasma parameter we first observe a collective shell rotation to occur, then, however, angular order is restored again before the cluster melts completely. We interpret the reentrant ordered state as a consequence of particle fluctuations in radial direction which increase the energy barrier for angular particle excursions in adjacent shells and thus stabilizes this excitation mode.

We gratefully acknowledge stimulating discussions with P. Maaß and financial support from the Deutsche Forschungsgemeinschaft (SFB 513).

[1] G. Birkl, S. Kassner, and H. Walther, Europhys. News 23, 143 (1992).

[2] See, e.g., 2D Electron Systems on He and Other Cryogenic Substrates, edited by E. Andrei (Kluwer Academic Publishers, Dordrecht, The Netherlands, 1998).

[3] P. Leiderer, J. Low Temp. Phys. 87, 247 (1992).

[4] M. A. Reed and W.P. Kirk, Nanostructure Physics and Fabrication (Academic Press, Boston, 1989).

[5] Y.E. Lozovik and V.A. Mandelshtam, Phys. Lett. A 145, 269 (1990).

[6] Y.E. Lozovik and V. A. Mandelshtam, Phys. Lett. A 165, 469 (1992).

[7] F.M. Peeters, V.A. Schweigert, and V.M. Bedanow, Physica (Amsterdam) 212B, 237 (1995).

[8] V. M. Bedanow and F. M. Peeters, Phys. Rev. B 49, 2667 (1994).

[9] V.A. Schweigert and F. M. Peeters, Phys. Rev. B 51, 7700 (1995).

[10] J. M. Kosterlitz and D. J. Thouless, J. Phys. C 6, 1181 (1973).

[11] D. R. Nelson and B. I. Halperin, Phys. Rev. B 19, 2457 (1979).

[12] A. H. Marcus and S. A. Rice, Phys. Rev. E 55, 637 (1997).

[13] K. J. Strandburg, Rev. Mod. Phys. 60, 161 (1988).

[14] Q.-H. Wei, C. Bechinger, D. Rudhardt, and P. Leiderer, Phys. Rev. Lett. 81, 2606 (1998).

[15] R. Bubeck, S. Neser, C. Bechinger, and P. Leiderer, Prog. Colloid Polym. Sci. 110, 41 (1998).

[16] K. Zahn, J. M. Mendez, and G. Maret, Phys. Rev. Lett. 79, 175 (1997). 